\title{
Polychlorinated Trityl Radicals for Dynamic Nuclear Polarization: The Role of Chlorine Nuclei
}

\author{
Juan Carlos Paniagua, ${ }^{b}$ Verónica Mugnaini, ${ }^{c, d}$ Cristina Gabellieri, ${ }^{a}$ Miguel Feliz, ${ }^{b}$ Nans Roques, ${ }^{c}$ Jaume \\ Veciana $^{*, c, d}$, and Miquel Pons $*, a, b$ \\ ${ }_{5}$ Received (in $\left.X X X, X X X\right)$ Xth $X X X X X X X X X 200 X$, Accepted Xth $X X X X X X X X X 200 X$ \\ First published on the web Xth $X X X X X X X X X 200 X$ \\ DOI: 10.1039/b000000x
}

Polychorinated trityl radicals bearing carboxilate substituents are water soluble persistent radicals that can be used for Dynamic Nuclear Polarization. In contrast to other trityl radicals, the

10 polarization mechanism differs from the classical solid effect. DFT calculations performed to rationalize this behaviour support the hypothesis that polarization is transferred from the unpaired electron to chlorine nuclei and from these to carbon by spin diffusion. The marked differences observed between neutral and anionic forms of the radical will be discussed.

\section{Introduction}

${ }_{15}$ One of the main limitations of NMR is its low sensitivity associated with a very small population difference between different spin states at room temperature and achievable magnetic fields. The gyromagnetic ratio of the electron is 2640 times higher than that of carbon 13 nuclei resulting in a 20 much larger polarization at the same temperature and magnetic field. The high electron polarization can be transferred to nuclei in the bulk of the sample by irradiating at the frequency of electronic spin resonances. This is the principle of Dynamic Nuclear Polarization (DNP). ${ }^{1}$ The non25 equilibrium nuclear polarization results in a much higher NMR sensitivity. In dissolution DNP experiments, enhanced nuclear polarization is generated at temperatures close to $1 \mathrm{~K}$ in a solid sample containing a stable radical acting as polarizing agent and the molecule of interest in a glassing 30 solvent. When the nuclear polarization has built up, the sample is quickly dissolved and shuttled to a conventional NMR instrument for observation. ${ }^{2}$ Fast transfer is essential since magnetization is lost by relaxation.

The choice of the radical is crucial to determine the 35 polarization transfer mechanism and the efficiency of the process. ${ }^{3}$ The main mechanisms for solid-state DNP are the thermal mixing/cross effects and the solid-effect. The former two mechanisms are operative when the width of the EPR line is larger than the nuclear frequency. The solid-effect is 40 usually observed when the homogeneous EPR line-width of the radical is smaller than the nuclear Larmor frequency, as in the case of trityl radicals such as OX63 (Scheme 1), whose persistency is related to the bulky substituents sterically hindering the central $\mathrm{sp}^{2}$ carbon where most of the spin 45 density resides. When this mechanism is operating, optimal enhancement is achieved by irradiating at frequencies separated from the electron resonance frequency by the nuclear frequency.

We have recently described the use of water soluble 50 polychlorotriphenylmethyl (PTM) radicals as polarizing agents in DNP experiments to enhance carbon-13 polarization. ${ }^{4}$ We have suggested that the electron polarization could be initially transferred intramolecularly to chlorine nuclei and that the observed carbon polarization 55 comes from the polarized chlorine nuclei via spin diffusion.

In this article we extend our results to include a comparison of radicals tri para carboxylic PTM (1) and its sodium salt (2); hexa meta carboxylic PTM (3) and its sodium salt (4) using DFT calculations as well as experiments.
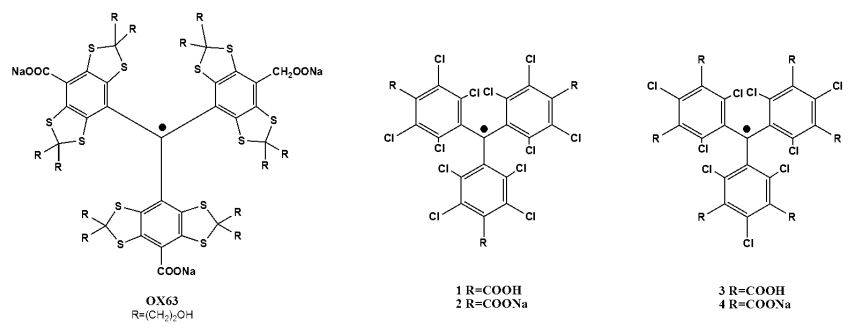

Scheme 1. Structures of OX63 and the investigated polychlorinated radicals.

65

All PTM radical derivatives show high stability and persistency thanks to their unique structure. The six ortho chlorine atoms are located at ca. $3 \AA$ with respect to the central $\mathrm{sp}^{2}$ carbon atom where most of the unpaired electron density is 70 expected. The sterical hindrance ensures the persistence and the chemical stability of the radicals. The para or meta positions of the chlorinated phenyl rings can be occupied by distinct functional group(s), hence modulating the supramolecular interactions of these trigonal molecules. Namely, these radicals 75 have been successfully used as robust open-shell organic synthons for the preparation of multidimensional networks showing magnetic ordering at low temperatures. ${ }^{5}$ The three phenyl rings assume a propeller-like conformation, with dihedral angles between the phenyl rings, as determined from 80 the crystal structures, similarly to the ones reported for OX63. ${ }^{6}$ This high out-of-plane torsion of the phenyl rings causes in these radicals a decrease in the conjugation of the $\pi$ electron system and hence affects the spin density distribution. 


\section{Experimental and computational methods}

Experimental methods. Substituted PTM radicals were synthesized as previosuly described. ${ }^{7}$ OX63 is a commercial 5 (Oxford Molecular Biotools) non-chlorinated trityl radical with carboxilate groups as sodium salts in the para position.

A Bruker Elexsys X-band instrument equipped with a rectangular standard cavity (TE 102) was used to record the EPR room temperature spectra.

${ }_{10}$ Ex-situ DNP polarisation was performed using a HyperSense ${ }^{\circledR}$ DNP polariser (Oxford Instruments, UK).

Frequency calibration was done using the sodium salt of the radicals dissolved at $15 \mathrm{mM}$ concentration in neat $1-{ }^{13} \mathrm{C}$ enriched pyruvic acid (Cortecnet). Pyruvic acid forms a glass 15 and, in its enriched form, gives enough sensitivity to carry out frequency sweeps by detecting the carbon signal in solid state. For transfer experiments, $1-{ }^{13} \mathrm{C}$ pyruvate sodium salt, $2-{ }^{13} \mathrm{C}$ acetone, or ${ }^{13} \mathrm{C}$ urea $\left(99 \%{ }^{13} \mathrm{C}\right.$ enriched, Sigma Aldrich) were dissolved in a $1 \mathrm{M}$ mixture of $\mathrm{H}_{2} \mathrm{O}$ :DMSO $(1: 1)$ containing 15

$20 \mathrm{mM}$ of the radical of choice, polarised for 1.5 hour at $1.4 \mathrm{~K}$ while irradiating $(100 \mathrm{~mW}$ power $)$ at the microwave frequency corresponding at the first maximum of the microwave sweep of the corresponding radical. The polarised sample was then rapidly dissolved in $4 \mathrm{ml}$ methanol and 25 transferred to a $5 \mathrm{~mm}$ tube placed inside the NMR spectrometer (500 MHz Varian Inova). A ${ }^{13} \mathrm{C}-\mathrm{NMR}$ spectrum of the polarised solution was recorded employing a $90^{\circ} \mathrm{RF}$ pulse. This whole process took around 4 seconds. After decay of the polarisation, a thermal equilibrium spectrum was 30 recorded for each sample (1024 transients) with a $20^{\circ}$ flip angle. Data were analyzed by using VNMRJ Varian and MestreNova software. The enhancement factor was calculated by the ratio of the polarised signal to the thermal equilibrium multiplied by the square root of the number of scans.

${ }_{35}$ Computational details. Relevant properties were calculated for the four radicals of Scheme 1 using Density Functional Theory (DFT). The hybrid exchange-correlation functional $\mathrm{B} \mathrm{LYP}^{8}$ was chosen, since it has proven to provide a rather accurate description of the electronic density at a much lower 40 cost than high level quantum chemical methods. ${ }^{9}$ In every case all-electron calculations have been performed so as to get the correct nodal properties of the orbitals in the core region. A crucial point for obtaining a reliable description of the core region with DFT methodology is to use a very fine quadrature 45 grid for the numerical calculation of integrals over functionals. We had to use three computational packages to obtain the desired properties, due to limitations of each of them. Since their implemented grid systems were not completely equivalent, large grids of similar type were 50 chosen. The reported Fermi contact couplings and spin dipole couplings were calculated with the Gaussian 09 rev. A.02 package $^{10}$ using the ultrafine grid (a pruned grid with 99 radial shells around each atom, and 590 angular points in each shell). ${ }^{11}$ The quadrupole coupling constant and g-tensors were 55 obtained with the Orca 2.7 rev. 0 program $^{12}$ using grid 6 (Lebedev with 590 angular points) for the SCF iterations and grid 7 (Lebedev with 770 angular points) for the final point, and the Gauss-Chebyshev radial integration method. ${ }^{13}$ The number of radial points is atom dependent, as specified in the ${ }_{60}$ user manual. In every case the tightest convergente option was chosen for the SCF convergence. The coupled-perturbed method has been used for evaluating the spin-orbit coupling. ${ }^{14}$ For obtaining starting symmetrized geometries and for some additional calculations the GAMESS ver. 12 Jan 2009 R3 ${ }_{65}$ package $^{15}$ has also been used with 590 angular and 96 radial points.

Preliminary calculations with a 6-31G(d,p) basis set were used to obtain reasonable starting geometries. ${ }^{16}$ This basis set has been designed to provide a good description of properties 70 dependent mostly on the valence region of the electron density. To calculate the hyperfine coupling constants, we used the IGLO basis set, ${ }^{17}$ specifically designed to reproduce such core properties and that covers all nuclei we are interested in. In order to check for convergence with the basis 75 set level, calculations were performed with both the IGLO-II and IGLO-III sets. Both sets gave very similar results, indicating a good degree of convergence. Only the results with the larger set will be shown. In every case molecular geometries were optimized with the same basis set used for 80 property calculations, so as to ensure that the electric field at the nuclei vanishes. Each radical has several conformations differing in the orientation of the rings and of the carboxyl groups. Some of them are symmetry equivalent and others have small energy differences. In these cases the most stable 85 ones have been chosen. Their symmetry point group is $C_{3}$ for radical 1 and $D_{3}$ for the others. ${ }^{18}$

\section{Results and discussion}

\section{3.1. X-band EPR in solution}

Room temperature X-band EPR spectra have been recorded for the chlorinated compounds $\mathbf{1 - 4}$ in a mixture of $\mathrm{H}_{2} \mathrm{O}$ :DMSO 1:1 (Fig. 1).

95

a

C
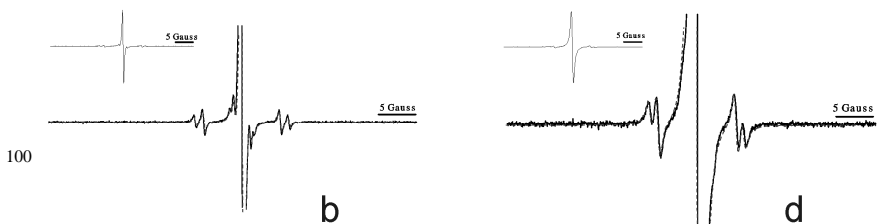

105
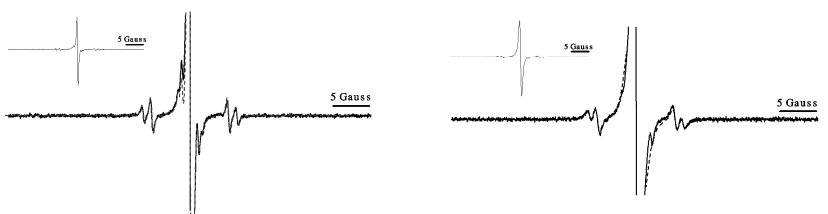

Figure 1. EPR X-band spectra in DMSO: $\mathrm{H}_{2} \mathrm{O} 1: 1$ at room temperature. a) 1; b) 2; c) 3; d) 4. Each simulated spectrum (dash gray line) is superimposed to the experimental one (solid black line) recorded at high MW power to see the ${ }^{13} \mathrm{C}$ 110 hyperfine couplings. In the inset the full range experimental spectrum recorded under optimal power conditions.

Narrow EPR linewidths (less than $0.7 \mathrm{G}$ in the absence of oxygen) are observed for all the chlorinated compounds. The $115 \mathrm{~g}$-values for $\mathbf{1}$ and $\mathbf{2}$ (with three carboxylic substituents, 
2.0029 and 2.0021 respectively) are lower than for $\mathbf{3}$ and $\mathbf{4}$ (with six carboxylic substituents, 2.0041 and 2.0034 respectively). ${ }^{19}$ As a reference the $g$-value of OX63 in $\mathrm{H}_{2} \mathrm{O}$ :DMSO 1:1 is 2.0031 and the EPR linewidth is $0.2 \mathrm{G}$.

${ }_{5}$ Thanks to the presence of large number of symmetry related carbons it is possible to measure the hyperfine couplings to carbon-13 nuclei at natural abundance, observed as satellite lines. The coupling to the central carbon could not be observed. This is probably due to fast relaxation because of

10 the slow tumbling rate of the molecule in this viscous solvent under the used experimental conditions. ${ }^{20}$ The largest observed coupling arise from the phenyl ${ }^{13} \mathrm{C}$ bridgehead (ipso), followed by the phenyl ${ }^{13} \mathrm{C}$ in ortho $(2,6)$ with respect to the central carbon atom, followed by the coupling with the ${ }^{15}$ phenyl ${ }^{13} \mathrm{C}$ in para $(4)$ and meta $(3,5)$ positions. Hyperfine couplings were accurately determined by simulation of the experimental spectra. ${ }^{21}$ Moreover, hyperfine couplings and $g$ values were computed for radicals 1-4 (see below). The experimental and calculated hyperfine couplings to carbon are 20 compared in table 1 .

Table 1. Experimental and calculated ${ }^{13} \mathrm{C}$-hyperfine coupling constants and $g$-values of PTM radicals 1-4 and OX63 in 1:1 25 DMSO: $\mathrm{H}_{2} \mathrm{O}$

\begin{tabular}{|c|c|c|c|c|c|c|}
\hline \multirow{2}{*}{\multicolumn{2}{|c|}{ Position: }} & \multicolumn{4}{|c|}{${ }^{13} \mathrm{C}$ Hyperfine couplings ${ }^{\mathrm{a}} / \mathrm{MHz}$} & \multirow[t]{2}{*}{$g$-value ${ }^{b}$} \\
\hline & & $\begin{array}{c}1 \\
\text { (ipso) }\end{array}$ & $\begin{array}{c}2,6 \\
(\text { ortho }\end{array}$ & $\begin{array}{c}3,5 \\
(\text { meta) }\end{array}$ & $\begin{array}{c}4 \\
(\operatorname{nar} a)\end{array}$ & \\
\hline \multirow{2}{*}{1} & exp.: & 35.76 & 29.00 & 5.88 & 8.68 & 2.0029 \\
\hline & calc.: & -34.43 & 29.65 & -6.14 & 7.74 & 2.0025 \\
\hline \multirow{2}{*}{2} & exp. : & 35.62 & 29.12 & 5.88 & 8.68 & 2.0021 \\
\hline & calc.: & -34.09 & 29.18 & -5.88 & 7.57 & 2.0026 \\
\hline \multirow{2}{*}{3} & exp.: & 35.42 & 29.68 & 5.94 & 8.96 & 2.0041 \\
\hline & calc.: & -34.33 & 30.79 & -5.94 & 9.12 & 2.0040 \\
\hline \multirow{2}{*}{4} & exp.: & 35.48 & 29.4 & 5.6 & 8.12 & 2.0034 \\
\hline & calc.: & -35.55 & 30.27 & -5.50 & 8.07 & 2.0035 \\
\hline \multirow{2}{*}{ OX63 } & exp. : & 31.36 & 25.06 & 6.16 & 9.24 & 2.0031 \\
\hline & calc.: ${ }^{\mathrm{c}}$ & -32.51 & 25.66 & -5.07 & 5.90 & - \\
\hline
\end{tabular}

${ }^{a}$ Hyperfine couplings are measured in absolute value. DFT calculations give also the sign. Experimental values were obtained by simulation of the experimental spectra including hyperfine couplings to the carbonyl carbons 30 that were equal or smaller than those to meta carbons.

b Experimental uncertainty is \pm 0.0003 .

c Ref.6

\section{3.2. DNP experiments}

As previously reported, ${ }^{4}$ carbon-13 DNP enhancements were observed using the sodium salts $\mathbf{2}$ and $\mathbf{4}$. However, there are substantial differences between PTM and other trityl radicals, 40 such as OX63.

The first one concerns the microwave frequencies at which optimal carbon-13 enhancements are observed. When the solid-effect mechanism is operative two maxima separated by $2 * v_{\mathrm{C}}$ are expected. Indeed, for OX63 a frequency separation 45 of $72 \mathrm{MHz}$ was observed, consistent with twice the Larmor frequency of carbon at $3.38 \mathrm{~T}$, using the radical dissolved in neat $1-{ }^{13} \mathrm{C}$-pyruvic acid at $1.5 \mathrm{~K}$. The separation of the two maxima for 2 and 4 in neat pyruvic acid was 124 and 136 $\mathrm{MHz}$, respectively, and the two maxima are broader. These 50 values indicate that carbons are not polarized by a direct solid-effect mechanism.

The second striking difference was observed in the case of $\mathbf{4}$. where negative or positive DNP enhancements were observed, for distinct substrates or even for different ${ }_{55}$ ionization states of the same substrate. ${ }^{4}$ This effect was shown to be a genuine DNP effect as the sign was reversed by changing the irradiation frequency from the lower to the higher frequency side of the electronic resonance frequency.

A third peculiarity, not previously reported, is the very ${ }_{60}$ different enhancements obtained with the neutral or anionic forms of the radicals. Only a broad profile was observed when polarization of ${ }^{13} \mathrm{C}$-pyruvic acid was attempted using the protonated radicals $\mathbf{1}$ and $\mathbf{3}$ (results not shown). Attempts to polarize carbon nuclei using the protonated forms of the 65 radicals and the frequencies optimized for their sodium salts resulted in weaker enhancements than with the sodium salts (Table 2).

Table 2. DNP enhancements after transfer ${ }^{\mathrm{a}}$

\begin{tabular}{|c|c|c|c|}
\hline & $1-{ }^{13} \mathrm{C}$ Pyruvate & ${ }^{13} \mathrm{C}$ Urea & $2-{ }^{13} \mathrm{C}$ Acetone \\
\hline OX63 $^{b}$ & 39214 & 12430 & 10863 \\
\hline $\mathbf{1}^{\mathrm{c}}$ & 198 & 2320 & 1509 \\
\hline $2^{b}$ & 29925 & 6392 & 4061 \\
\hline $3^{\mathrm{c}}$ & 1780 & 1891 & 517 \\
\hline $4^{b}$ & 3026 & 2545 & 901 \\
\hline \multicolumn{4}{|c|}{$\begin{array}{l}\text { a Enhancements were calculated as described in the experimental sectio } \\
\text { b }\end{array}$} \\
\hline
\end{tabular}

75 In order to try to rationalize the origin of the singular DNP effects observed with PTM radicals, we have carried out detailed DFT calculations of the hyperfine couplings in four PTM radicals with distinct chlorine substituents and different ionization states.

80

\subsection{DFT calculations}

Figure 2 shows the optimized geometries and spin densities of radicals 1-4 computed using DFT and IGLO-III basis set.

${ }_{85}$ Table 1 shows a comparison of calculated and experimental $g$ values and isotropic hyperfine couplings to carbons for PTM radicals. As a reference, the experimental values of OX63 measured by us in the same conditions are compared with the calculated values in reference 6 .

90 DFT calculations accurately predict the different $g$-values of the the two families of PTM radicals. Hyperfine couplings are also well reproduced for all the aromatic carbons. 


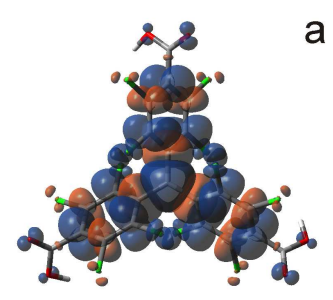

a
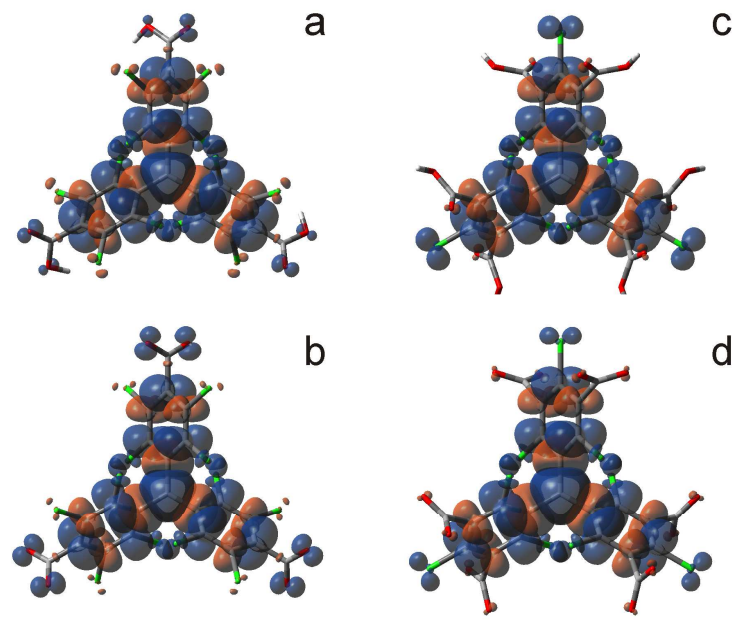

Figure 2. Optimized geometries and spin densities of PTM radicals. a) 1; b) 2; c) 3 ; d) 4 . The largest spin density in chlorine atoms is in the para position of radical 3, which also gives the largest hyperfine coupling. The isodensity I surface has been drawn at 0.0005 a.u.

The good agreement between the calculated and experimental $g$-values and observed hyperfine couplings with carbon 13 provide a validation for the calculations. Hyperfine couplings 10 with chlorine atoms are not observed in solution due to the fast relaxation of quadrupolar chlorine nuclei. However substantial couplings are predicted by the calculations. The calculated isotropic Fermi coupling and the principal components of the dipolar coupling tensor (including the 15 isotropic and anisotropic terms) are given in Table 2. The anisotropic coupling is relevant since DNP is carried out in a solid glass sample.

Table 3 Calculated chlorine hyperfine couplings in PTM 20 radicals $\mathbf{1 - 4}$

\begin{tabular}{|c|c|c|c|c|c|}
\hline \multirow{3}{*}{1} & & Fermi/MHz & $\mathrm{D}_{11} / \mathrm{MHz}$ & $\mathrm{D}_{22} / \mathrm{MHz}$ & $\mathrm{D}_{33} / \mathrm{MHz}$ \\
\hline & $\mathrm{Cl}_{\text {ortho }}$ & 0.35 & -2.43 & -0.41 & 3.19 \\
\hline & $\mathrm{Cl}_{\text {meta }}$ & 0.15 & -1.98 & 0.44 & 1.69 \\
\hline \multirow{2}{*}{2} & $\mathrm{Cl}_{\text {ortho }}$ & 0.09 & -2.11 & -0.23 & 2.43 \\
\hline & $\mathrm{Cl}_{\text {meta }}$ & 0.17 & -1.62 & 0.30 & 1.50 \\
\hline \multirow{2}{*}{3} & $\mathrm{Cl}_{\text {ortho }}$ & 0.24 & -2.22 & -0.37 & 2.83 \\
\hline & $\mathrm{Cl}_{\text {para }}$ & 0.78 & -2.96 & -1.85 & 5.59 \\
\hline \multirow{2}{*}{4} & $\mathrm{Cl}_{\text {ortho }}$ & 0.33 & -1.95 & -0.06 & 2.34 \\
\hline & $\mathrm{Cl}_{\text {para }}$ & 0.3 & -1.83 & -0.92 & 3.05 \\
\hline
\end{tabular}

The calculated isotropic hyperfine couplings to chlorine atoms 25 are larger in the ortho and para positions than in meta, except for 2 where the Fermi coupling to the ortho chlorine is smaller. The calculated couplings to chlorine nuclei correlate well with the spin densities around these atoms shown in Figure 2. The unpaired spin densities near the para chlorine
30 atoms in radicals $\mathbf{3}$ and $\mathbf{4}$ are much higher than those in the meta chlorine atoms of $\mathbf{1}$ and $\mathbf{2}$. Also the spin densities in the para chlorines is larger in $\mathbf{3}$ than in $\mathbf{4}$. When the anisotropic coupling is considered, the distribution of values of the three principal components of the chlorine hyperfine coupling 35 tensor in radicals $\mathbf{2}$ and $\mathbf{4}$ are very different. In the case of $\mathbf{2}$ for both the ortho and meta chlorine nuclei, the mean of $\mathrm{D}_{11}$ and $\mathrm{D}_{33}$ is close to $\mathrm{D}_{22}$, i.e. the expected powder pattern of the hyperfine coupling to both chlorine atoms is rather symmetrical. In contrast, in the case of the para chlorine of $\mathbf{4}$,

40 the mean of $\mathrm{D}_{11}$ and $\mathrm{D}_{33}(+0.61 \mathrm{MHz})$ is substantially different from $\mathrm{D}_{22}(-0.92 \mathrm{MHz})$, indicating a very asymmetric powder pattern. Interestingly, only for radical 4 negative DNP enhancements have been observed.

When equivalent chlorine atoms of the free acids and the 45 corresponding salts are compared, the three principal components are larger in magnitude in the free acids than in the sodium salts. However, the same trends concerning the symmetry of the powder distribution are observed for the free acids and the salts. In the hexacarboxylic radicals $\mathbf{3}$ and $\mathbf{4}$, 50 coupling to the para chlorine atoms is predicted to be larger than to those in ortho, in spite of a much larger distance to the $\mathrm{sp}^{2}$ central carbon.

The calculations reveal that the large number of magnetically 55 active, spin 3/2, chlorine nuclei located in the periphery of PTM radicals are effectively coupled to the unpaired electron and could be effectively polarized by irradiation at the proper microwave frequency. The chlorine nuclei could subsequently act as a source of polarization of other nuclei in the sample.

60

The reason for the marked difference between the protonated and anionic forms of the radicals is not obvious. Chlorine relaxation is expected to be slow in the solid sample. Short range magnetic ordering may result in a longer correlation 65 time for electron exchange and may provide an additional relaxation mechanism for chlorine, which could quench DNP. Broadening of the NQR signal in $\mathrm{p}-\mathrm{Cl}$ BDPA radical has been reported at temperatures below $3.2 \mathrm{~K} .{ }^{22}$

Intermolecular hydrogen bond formation is possible in the 70 acids $\mathbf{1}$ and $\mathbf{3}$ but not in the salts. Previous observations from some of us showed that crystals of $\mathbf{3}$ show magnetic interactions (ferromagnetic or antiferromagnetic, depending on the solvate) arising from hydrogen bonded contacts between radicals. 5,23

75

The Larmor frequency at $3.38 \mathrm{~T}$ of ${ }^{35} \mathrm{Cl}$ is $14.1 \mathrm{MHz}$ and that of ${ }^{37} \mathrm{Cl}$ is $11.7 \mathrm{MHz}$. Both isotopes have large quadrupolar moments of $-8.2 \times 10^{-30} \mathrm{~m}^{2}$ and $-6.4 \times 10^{-30} \mathrm{~m}^{2}$, respectively. Quadrupolar couplings of aromatic chlorinated molecules are 80 typically around $-70 \mathrm{MHz},{ }^{24}$ larger than the Zeeman chlorine frequency but neither interaction can be considered just as a perturbation of the other one. Under these conditions, multiple quantum transitions become allowed. Direct observation of forbidden transitions has been made in an oriented sodium 85 chlorate single crystal and confirmed by exact calculations of the transition frequencies. ${ }^{25}$ When dipolar couplings between spin $1 / 2$ and quadrupolar nuclei are considered, one should 
keep in mind that the quantization axes of chlorine and $1 / 2$ spins are not necessarily collinear. ${ }^{26}$ The effect of quadrupolar nuclei in polycrystalline EPR spectra was calculated by Rollmann and Chan. ${ }^{27}$ They noticed that transitions implying ${ }_{5}|\Delta \mathrm{m}|>1$ were important in these systems.

For a cylindrically symmetric system in which the quadrupolar and Zeeman axes of chlorine were collinear, the transition frequencies would include simple additive combinations of the Zeeman multiple quantum terms and the 10 quadrupolar frequencies. For other orientations the frequencies depend on the angle of the Zeeman and quadrupolar terms. Interestingly, the order of magnitude of the separation of the two microwave frequency maxima that give optimal DNP to carbons coincide with twice the 15 frequency of the double quantum ${ }^{35} \mathrm{Cl}$ transition plus the expected isotropic quadrupolar energy splitting. With quadrupolar couplings in the range of $-70 \mathrm{MHz}$ to $-80 \mathrm{MHz}$, the expected separations would be $126 \mathrm{MHz}$ to $136 \mathrm{MHz}$, very close to the $124 \mathrm{MHz}$ and $136 \mathrm{MHz}$ observed in the case of 2 20 and 4 in neat pyruvic acid. The exact energy differences will depend on the orientation of the radical in the glass. A more detailed analysis, taking into account this orientational dependence is beyond the scope of this article but a broad maximum is expected.

\section{${ }_{25}$ Conclusions}

Chlorinated trityl radicals are chemically stable and give narrow EPR lines in solution. DNP enhancement of carbon-13 nuclei has been demonstrated using polychlorinated 30 derivatives 1-4, differing in the position of chlorine atoms and of carboxylate/carboxylic groups. Maximum DNP enhancements are obtained with the sodium salts of the radicals. PTM derivative $\mathbf{2}$ shows a DNP efficiency comparable, although slightly lower, to the widely used OX63

35 radical. Derivative $\mathbf{4}$ has a lower efficiency but it shows striking supramolecular effects that may shed light on the details of the initial polarization transfer step with different substrates.

The microwave frequencies that give the maximum 40 enhancement do not support the solid effect mechanism of other non-chlorinated trityl radicals. Instead, they suggest that carbon-13 polarization takes place by internuclear polarization from chlorine nuclei that are polarized by the electron through hyperfine coupling.

${ }_{45}$ DFT calculations correctly reproduce the EPR $g$-values and hyperfine couplings to carbons that can be measured experimentally. Moreover they are able to supply the values of the hyperfine couplings to chlorines. These are large and dramatically sensitive to position as well as to the ionization 50 state of the substituent. The para chlorine nuclei in $\mathbf{3}$ and $\mathbf{4}$ have the largest couplings, in spite of being the more distant to the centre of the radical, where most of the unpaired spin density is located. Hyperfine couplings predicted by the calculations show that there is an efficient way to 55 hyperpolarize chlorine nuclei. The degree of polarization will also depend on the efficiency of the competing relaxation pathways. The relatively large paramagnetic density in the peripheral chlorine nuclei may contribute to relaxation, specially if intermolecular interactions are favoured, e.g. by 60 hydrogen bonding. This may contribute to the observed differences in the enhancements obtained with the tri para carboxylate (2) and hexa meta carboxylate PTMs (4) salts and also between these and their protonated forms ( $\mathbf{1}$ and $\mathbf{3})$.

Further experimental and theoretical work is in progress to try ${ }_{65}$ to advance towards the quantitative prediction of the observed effects.

One of the predictions of the indirect polarization model is that it should be short range, due to the low gyromagnetic ratio of chlorine, affecting only those nuclei that are close to 70 the chlorine atoms in the glass. The fact that polarization takes place via chlorine nuclei implies that the irradiation frequency to enhance distinct nuclei would be the same. This may open the possibility of using chlorinated trityl groups as universal radicals for multinuclear applications or to enhance 75 the sensitivity of low frequency nuclei.

\section{Acknowledgments}

We thank Dr. Maria Antonia Molins for support in the 80 operation and maintenance of the Hypersense instrument, Malena Oliveros for the synthesis of the chlorinated radicals 1 and 2 and Dr. F. Mota for his help in solving computational problems. This work was partially supported by the MICINNSpain, (BIO2007-63458, Project Consolider-C ${ }_{85}$ EMOCIONa,CTQ2006-06333/BQU, and LRB-ICTS), from the Generalitat de Catalunya, (2009SGR516 and 2009SGR1352) and from the Networking Research Center on Bioengineering, Biomaterials, and NanoMedicine (CIBERBBN), promoted by ISCIII, Spain. V.M. acknowledges the 90 MICINN-Spain for a Juan de la Cierva postdoctoral contract. Preliminary data were acquired in Birmingham with support from EU-NMR. Computational facilities provided by the Centre de Supercomputació de Catalunya (CESCA) through a grant from the Universitat de Barcelona are acknowledged. 95

\section{Notes and references}

${ }^{a}$ Institute for Research in Biomedicine, Parc Científic de Barcelona, Baldiri Reixac, 10-12 08028-Barcelona, Spain . Fax: +34934039976; 100 Tel: +34934034683; E-mail: mpons@ub.edu.

${ }^{b}$ Universitat de Barcelona. Martí i Franquès 1-11 08028 Barcelona, Spain.

${ }^{c}$ Institute of Materials Science of Barcelona (ICMAB-CSIC), Bellaterra, Spain.E-mail: vecianaj@icmab.es.

${ }_{105}{ }^{d}$ Networking Research Center on Bioengineering, Biomaterials, and

NanoMedicine (CIBER-BBN), Bellaterra, Spain,

1 T. R.Carver, C. P. Slichter, Phys. Rev. 1953, 92, 212; A. Abragam, M.Goldman, Rep. Prog. Phys. 1978, 41, 395.

2 J. H. Ardenkjær-Larsen, F. Björn, A. Gram, G. Hansson, L. Hansson, M.H. Lerche, R. Servin, M. Thaning, K. Golman, Proc. Natl. Acad. Sci. U.S.A. 2003, 100, 10158; A.-H. Emwas, M. Saunders, C. Ludwig, U. L. Günther, Appl. Magn. Res., 2008,34,483.

3 Th. Maly, G. T. Debelouchina, V. S. Bajaj, K-N. Hu, C-G. Joo, M. L. Mak-Jurkauskas, J. R. Sirigiri, P. C. A. van der Wel, J. Herzfeld, R. J. Temkin, R. G. Griffin J. Chem. Phys. 2008, 128, 052211. 
4 C. Gabellieri, V. Mugnaini, J. C. Paniagua, N. Roques, M. Oliveros, M. Feliz, J. Veciana, M. Pons, Angew. Chem. Int. Ed. In press.

5 D. Maspoch, N. Domingo, N. Roques, K. Wurst, J. Tejada, C. Rovira, D. Ruiz-Molina,. J. Veciana, Chem. Eur. J. 2007, 13, 8153; N. Roques, D. Maspoch, A. Datcu, K. Wurst, D. Ruiz-Molina, C. Rovira, J. Veciana, Polyhedron 2007, 26, 1934; D. Maspoch, D. Ruiz-Molina, K. Wurst, N. Domingo, M. Cavallini, F. Biscarini, J, Tejada, C. Rovira, J. Veciana, Nature Mat., 2003, 190.

6 M. K. Bowman, C. Mailer, H.J. Halpern, J. Magn. Res. 2005, 172 , 254.

7 D. Maspoch, N. Domingo, D. Ruiz-Molina, K. Wurst, G. Vanghan, J. Tejada, C. Rovira, J. Veciana, Angew. Chem. Int. Ed. 2004, 43, 1828; N. Roques, D. Maspoch, K. Wurst, D. Ruiz-Molina, C. Rovira, J. Veciana, Chem. Eur. J. 2006, 12, 9238.

8 A. D. Becke, J. Chem. Phys. 1993, 98, 5648; C. Lee, W. Yang and R. G. Parr, Phys. Rev. B 1988, 37, 785.

9 P. Schwerdtfeger, M. Pernpointner and J. K. Laerdahl, J. Chem. Phys. 1999, 111, 3357; A. Rizzo, K. Ruud, T. Helgaker, P. Salek, H. Ågren, O. Vahtras, Chem. Phys. Let. 2003, 372, 377.

10 Gaussian 09, revision A.02, M. J. Frisch, G. W. Trucks, H. B. Schlegel, G. E. Scuseria, M. A. Robb, J. R. Cheeseman, G. Scalmani, V. Barone, B. Mennucci, G. A. Petersson, H. Nakatsuji, M. Caricato, X. Li, H. P. Hratchian, A. F. Izmaylov, J. Bloino, G. Zheng, J. L. Sonnenberg, M. Hada, M. Ehara, K. Toyota, R. Fukuda, J. Hasegawa, M. Ishida, T. Nakajima, Y. Honda, O. Kitao, H. Nakai, T. Vreven, J. A. Montgomery, Jr., J. E. Peralta, F. Ogliaro, M. Bearpark, J. J. Heyd, E. Brothers, K. N. Kudin, V. N. Staroverov, R. Kobayashi, J. Normand, K. Raghavachari, A. Rendell, J. C. Burant, S. S. Iyengar, J. Tomasi, M. Cossi, N. Rega, J. M. Millam, M. Klene, J. E. Knox, J. B. Cross, V. Bakken, C. Adamo, J. Jaramillo, R. Gomperts, R. E. Stratmann, O. Yazyev, A. J. Austin, R. Cammi, C. Pomelli, J. W. Ochterski, R. L. Martin, K. Morokuma, V. G. Zakrzewski, G. A. Voth, P. Salvador, J. J. Dannenberg, S. Dapprich, A. D. Daniels, O. Farkas, J. B. Foresman, J. V. Ortiz, J. Cioslowski, and D. J. Fox, Gaussian, Inc., Wallingford CT, 2009

11 V. I. Lebedev and L. Skorokhodov, Russian Acad. Sci. Dokl. Math. 1992, 45, 587.

12 ORCA version 2.7 rev. 0, F. Neese, Lehrstuhl fuer Theoretische Chemie, Institut fuer Physikalische und Theoretische Chemie, Universitaet Bonn, Germany, June 2009.

13 O. Treutler, R. Ahlrichs, J. Chem. Phys. 1994, 102, 346; M. Krack, A. M. Köster, J. Chem. Phys. 1998, 108, 3226.

14 F. Neese, J. Chem. Phys, 2007, 127, 164112.

15 M. W. Schmidt, K. K. Baldridge, J. A. Boatz, S. T. Elbert, M. S. Gordon, J. J. Jensen, S. Koseki, N. Matsunaga, K. A. Nguyen, S. Su, T. L. Windus, M. Dupuis, J. A. Montgomery, J. Comput. Chem. 1993, 14, 1347; M. S. Gordon, M. W. Schmidt, in Theory and Applications of Computational Chemistry, the first forty years, C. E. Dykstra, G. Frenking, K. S. Kim, G. E. Scuseria (editors), Elsevier, 2005, p 1167.

16 W. J. Hehre, R. Ditchfield and J. A. Pople, J. Chem. Phys. 1972, 56, 2257; P. C. Hariharan and J. A. Pople, Theoret. Chimica Acta 1973, 28, 213; M. M. Francl, W. J. Petro, W. J. Hehre, J. S. Binkley, M. S. Gordon, D. J. DeFrees and J. A. Pople, J. Chem. Phys. 1982, 77, 3654 .

17 W. Kutzelnigg, U. Fleischer, M. Schindler, The IGLO-Method: Ab Initio Calculation and Interpretation of NMR Chemical Shifts and Magnetic Susceptibilities, Springer-Verlag, 1990, vol. 23.

18 The lower symmetry of $\mathbf{1}$ with respect to $\mathbf{2}$ is due to the presence of the proton in the carboxylic acid. Slightly different values are obtained by the two ortho and meta carbons of $\mathbf{1}$. The reported values in table 1 are average values.

19 The uncertainty of $g$-values measured from CW X-band spectra is \pm 0.0003 .

20 For other PTM derivatives, the coupling with the central carbon atom has been found to be around $30 \mathrm{G}(\mathrm{O}$. Armet, J. Veciana, C. Rovira, J. Riera, J. Castañer, E. Molins, J. Rius, C. Miravitlles, S Olivella, J. Brichfeus, J. Phys. Chem. 1987, 91, 5608). The reported coupling for OX63 is $23.96 \mathrm{G}$

21 Simulations made using SimFonia (Bruker).
22 T. Yoshioka, J. Yamauchi, H. Ohya-Nishiguchi, Y. Deguchi, Bull. Chem.Soc. Japan, 1975, 48, 335.

23 N. Roques, D. Maspoch,N. Domingo,D. Ruiz-Molina, K. Wurst, J. Tejada, C. Rovira, J. Veciana Chem. Commun., 2005, 4801.

24 E.A.C. Lucken, Nuclear Quadrupole Coupling Constants. Academic Press. London 1969; p 187

25 A.D. Bain, Mol Phys., 2003, 101, 3163; M.Khasawneh, J.S. Hartman, A.D. Bain, Mol.Phys, 2004, 102, 975.

26 D.L. VanderHart, H.S. Gutowsky, T.C. Farrar, J. Am. Chem. Soc. 1967, 89, 5056; J.G. Hexem, M.H. Frey, S.J. Opella, J. Chem. Phys. 1982, 77, 3847..

27 L.D. Rollmann, S.I. Chan, J. Chem. Phys, 1969, 50, 3416. 